\title{
Eléments pour une histoire de la communauté thérapeutique dans la psychiatrie occidentale de la seconde moitié du $20^{\mathrm{e}}$ siècle
}

Catherine Fussinger

\section{Summary}

Based on a critique of the traditional ruling of mental hospital, therapeutic community is an innovative model elaborated in Great Britain during World War II. According to this approach, all the relationships at work inside the institution have a big impact on the patients' state. One of the favoured tools of the therapeutic community lies in regular meetings common to patients and staff, but also reserved to professionals. During these sessions small and big problems are intended to be discussed and resolved collectively. The constitution of this approach as a model and its diffusion in continental European psychiatry during the second half of the 20th century is described in this paper. Four stages are distinguished: the genesis, the constitution of a distinct approach and diffusion in Continental Europe, the radicalisation and criticism by the antipsychiatric movement, the institutionalisation and decline.

Keywords: therapeutic community; 20th century psychiatry; Europe; psychosocial reform; antipsychiatry

\section{Résumé}

Basée sur une critique du système traditionnel de prise en charge des patient-e-s dans les hôpitaux psychiatriques, la communauté thérapeutique est un modèle novateur élaboré en Grande-Bretagne durant la Seconde Guerre mondiale, dans lequel l'ensemble des rapports interpersonnels à l'œuvre au sein de l'institution sont perçus comme ayant un fort impact

Catherine Fussinger, Institut universitaire d'histoire de la médecine et de la santé publique, 1, chemin des Falaises, CH-1005 Lausanne (Catherine.Fussinger@chuv.ch). 
sur l'état des malades. Aussi, l'un des instruments privilégiés de la communauté thérapeutique réside-t-il dans un système de réunions régulières entre soignant-e-s et soigné-e-s, mais aussi entre professionnel-le-s, au cours desquels petits et grands problèmes doivent être débattus et résolus collectivement. La constitution de cette approche comme un courant à part entière et sa diffusion au sein de la psychiatrie occidentale au cours de la seconde moitié du $20^{\text {ème }}$ siècle est retracée dans cet article en opérant une distinction entre quatre étapes: genèse, constitution du courant et diffusion en Europe continentale, appropriation et critique au sein du mouvement antipsychiatrique, institutionnalisation et déclin.

\section{Introduction}

Comme bon nombre de notions ayant eu cours au sein de la psychiatrie occidentale, la communauté thérapeutique s'avère difficile à appréhender. Si cela fut vrai dans les années 1950 à 1980, y compris dans le milieu psychiatrique, cela l'est davantage encore aujourd'hui. En effet, bien que ce modèle soit encore appliqué ici et là, il a cessé d'être investi comme une véritable alternative dans les institutions prenant en charge des personnes souffrant de troubles psychiques ${ }^{1}$.

Pourtant, après la première utilisation du terme en 1946, la communauté thérapeutique est rapidement devenue un modèle dont la carrière a duré quarante ans au moins. De plus, bien que son essor ait été au départ très étroitement lié à la psychiatrie britannique, puis nord-américaine, des communautés thérapeutiques furent créées dans nombre de pays occidentaux à partir du milieu des années 1960. La visibilité de ce courant demeura toutefois relative. En effet, si la mise en œuvre de cette approche au sein d'un hôpital psychiatrique exigeait un intense travail de négociations, ces discussions se déroulèrent essentiellement au niveau local. Elles n'impliquèrent donc pas de mobilisation d'ampleur nationale au sein de la profession psychiatrique, comme ce fut le cas pour d'autres approches novatrices en matière de politique de santé mentale. Cette inscription locale ainsi que la durée de vie souvent éphémère des expériences engagées expliquent sans doute pourquoi la communauté thérapeutique constitue un épisode peu connu de l'histoire de la psychiatrie dans la seconde moitié du $20^{\text {ème }}$ siècle - période qui, rappelonsle, demeure encore actuellement la moins étudiée en regard des 150 années précédentes.

1 Ce modèle reste par contre plus prégnant dans la prise en charge de personnes toxicomanes. 
L'historien-ne amené-e à constater le rôle joué par cette approche dans telle ou telle institution psychiatrique risque dès lors d'être confronté-e à plusieurs difficultés. Comme nous le verrons plus loin, la communauté thérapeutique est un «objet mou» reposant sur des définitions labiles. Mais surtout, dans la plupart des cas, sa mise en œuvre au niveau local ne s'est pas accompagnée de production de documents et le recours à l'histoire orale ne suffit pas toujours à pallier ce manque de sources écrites. De plus, la littérature secondaire ne permet que partiellement de surmonter ces difficultés. Certes, la plupart des ouvrages traitant de l'évolution de la psychiatrie au $20^{\text {ème }}$ siècle en Grande-Bretagne ou aux USA mentionnent la communauté thérapeutique, mais ils la présentent en quelques paragraphes seulement ${ }^{2}$. Quant aux travaux consacrés à ce sujet, ils portent essentiellement sur les prémisses de ce courant durant la guerre ${ }^{3}$, ainsi que sur ses promoteurs les plus connus ${ }^{4}$; quelques études s'emploient également à analyser des expériences locales. 5 Enfin, la communauté thérapeutique a suscité très tôt l'intérêt des sociologues et des ethnologues qui l'ont pour la plupart abordée à partir de la thèse du contrôle social ${ }^{6}$. Effectuées entre les années 1950 et 1980, ces recherches, d'un apport incontestable, sont toutefois susceptibles d'être classées aussi bien parmi les sources que dans la littérature secondaire ${ }^{7}$. Force est donc de constater l'absence de travaux historiques embrassant la carrière de la communauté thérapeutique dans son ensemble ${ }^{8}$. En retraçant les grandes étapes de son évolution, le présent article entend offrir des jalons à partir desquels des expériences singulières devraient être plus faciles à situer.

2 Jones 1972; Busfield 1986; Grob 1994.

3 Rose 1989; Jones 2004; Thalassis 2007; Harrison 2000.

4 Millard 1996; Harrison 2000.

5 Sur le rôle de la communauté thérapeutique au sein d'un hôpital de jour londonien: Spandler 2006. Dans sa thèse sur le mouvement antipsychiatrique aux Pays-Bas, Gemma Blok examine le fonctionnement d'une communauté thérapeutique établie dans un hôpital psychiatrique néerlandais: Blok 2004.

6 Volontiers qualifiée de foucaldienne aujourd'hui, cette perspective a de fait diverses sources d'inspiration. Pour le sociologue Michael Bloor (1986), dans le cas de la communauté thérapeutique, elle peut soit être appliquée au niveau macro (les finalités réadaptatives de la communauté thérapeutique se trouvent alors soulignées), soit au niveau micro (l'examen porte alors les rapports de pouvoir se jouant au quotidien). Victor Sharp (1975) aurait été le premier à étudier la communauté thérapeutique sous l'angle du contrôle social.

7 Suite à une première recherche menée dans la deuxième moitié des années 1950 (Rapoport 1960,1974) destinée à aider les psychiatres à mieux théoriser leur pratique, plusieurs autres études furent réalisées au cours des années 1970 et 80 dans une perspective qui se voulait plus critique (Sharp 1975; Bloor et al. 1982, 1986, 1988; Wiley 1986; McKeganey/Bloor 1987; Manning 1976, 1989).

8 Les sociologues Bloor, McKeganey, et Fonkert (1988), ainsi que Manning $(1976,1989)$ proposent de brefs historiques. Pour le point de vue de promoteurs de la communauté thérapeutique: Clark 1977; Campling/Haigh 1999. 
L'histoire de la communauté thérapeutique peut être divisée en quatre phases. Le premier moment, quelque peu mythique, est celui des expériences fondatrices au cours de la Seconde Guerre mondiale. La seconde étape, de 1945 au milieu des années 1960, voit la constitution à proprement parler de ce courant à travers la multiplication des expériences au sein des pays anglosaxons ainsi que leur théorisation dans diverses publications. La troisième phase, qui court du milieu des années 1960 à la fin des années 1970, comprend deux mouvements distincts. On assiste d'une part à une diffusion du modèle de la communauté thérapeutique dans plusieurs pays occidentaux. On observe d'autre part une appropriation de cette approche par le mouvement antipsychiatrique, dont il résulta une radicalisation de certaines pratiques mais aussi une remise en cause. A partir des années 1980 enfin, ce courant donne des signes d'institutionnalisation, les efforts déployés en ce sens ne semblent toutefois pas être parvenus à enrayer son déclin.

\section{La communauté thérapeutique: un premier portrait}

Avant de retracer la trajectoire de la communauté thérapeutique, il importe de commencer par en dresser un portrait plus précis. En concurrence avec des termes apparus à la même époque - tels que «psychiatrie sociale», «psychothérapie de groupe», mais aussi «psychiatrie administrative»-, la communauté thérapeutique fut d'emblée une notion élastique, ainsi que le relevèrent plusieurs acteurs sociaux $x^{9}$. Les différentes conceptions de cette approche ont toutefois en commun l'attention accordée à l'impact de «l'environnement social» sur l'état des patient-e-s. En effet, l'idée forte qui soustend la communauté thérapeutique est que si les règles de vie et l'ensemble des relations interpersonnelles à l'œuvre au sein des institutions psychiatriques s'avèrent en certains cas pathogènes, elles ont aussi un pouvoir thérapeutique.

L'emprise croissante des conceptions psychanalytiques dans les années 1950 explique en partie l'importance accordée à cette composante relationnelle. Cela dit, tous les promoteurs de la communauté thérapeutique ne se définissent pas comme psychanalystes et la plupart d'entre eux ont refusé de présenter ce courant comme une forme de psychothérapie à proprement parler. De plus, ils insistèrent souvent davantage sur la dimension «sociale» de leur approche ${ }^{10}$. Dans la mesure où ce terme renvoie

9 Bloch 1962,234sq.; Clark 1964, 42sq.; Clark 1977, 553; Bleandonu 1970, 9sq.; Main 1983, 217.

10 Clark $(1977,54)$ affirme par exemple que «l'analyse sociale» constitue l'outil principal de la communauté thérapeutique. 
surtout à la prise en compte des rapports interpersonnels et à la dynamique de groupe, le «social» tend toutefois à se confondre avec le «psychologique» dans ce contexte.

L'élaboration de la communauté thérapeutique s'inscrit dans le cadre des efforts déployés dès les années 1950 pour rompre avec le fonctionnement traditionnel des hôpitaux psychiatriques, dénoncé comme profondément aliénant pour les malades. Formulée par une frange de psychiatres réformateurs ${ }^{11}$, cette critique fut très vite à l'origine d'expériences alternatives. Partie prenante de ce mouvement, la communauté thérapeutique a ainsi parfois pu englober presque l'intégralité des réformes entreprises pour «humaniser» les hôpitaux psychiatriques (extension et diversification des activités occupationnelles et de réhabilitation; introduction de la mixité; tentative de rétablissement des liens avec l'extérieur par le biais de sorties en ville, de vacances et d'un maintien des contacts avec la famille; restitution des objets personnels; diminution ou suppression des pratiques d'enfermement et de contention, etc.). Si les théoriciens de la communauté thérapeutique situèrent bien leur travail dans le cadre de ces réformes, ils se distinguèrent cependant par l'attention particulière accordée à l'amélioration des relations et de la communication entre les malades et les soignant-e-s, mais aussi entre les différentes catégories de soignant-e-s.

De manière plus restrictive, la notion de communauté thérapeutique a pu être utilisée pour désigner le fonctionnement d'un petit groupe de malades (généralement entre 20 et 30 ) et de soignant-e-s adoptant des règles de vie en commun qui se voulaient en elles-mêmes thérapeutiques. De telles communautés furent parfois créées au sein d'une division d'un hôpital psychiatrique dans le cadre de projets pilotes, comme elles purent s'installer dans une maison individuelle dans une zone d'habitation ordinaire et se doter d'un statut institutionnel autonome (fondation, association) ${ }^{12}$.

Dans un cas comme dans l'autre, on retrouve cependant certaines caractéristiques communes dans ce qui se voulait une transformation radicale de l'organisation hiérarchique des institutions psychiatriques. Des réunions journalières, ou au moins pluri-hebdomadaires, réunissant l'ensemble des malades et du personnel constituent le trait le plus saillant du fonctionnement des communautés thérapeutiques. Basées sur une libre circulation

11 Parmi les ouvrages ayant eu beaucoup de retentissement, on peut citer Stanton Alfred \& Schwartz Morris, The Mental Hospital. A study of Institutional Participation in Psychiatric Illness and Treatment, 1954 et Barton Russel, Institutional Neurosis, 1959.

12 Initialement conçu à l'intention des patient-e-s psychiatriques, ce modèle a par la suite également été préconisé pour la prise en charge de personnes dépendantes (alcool, drogue), délinquantes ou emprisonnées; ces développements ne seront toutefois pas abordés dans cet article. 
de la parole, qualifiée à l'époque de «démocratique», ces rencontres étaient censées permettre d'aborder tous les problèmes et incidents survenus au sein de l'institution, ainsi que de formuler des propositions afin d'améliorer l'organisation de la vie communautaire ${ }^{13}$. Ce nouvel agencement avait pour but explicite d'amener le personnel et les patient-e-s à s'investir activement dans l'entreprise thérapeutique. Cela impliquait notamment, pour le personnel, de se déprendre d'une attitude décrite comme autoritaire, voire répressive, mais aussi d'accepter d'être régulièrement remis en cause lors des discussions collectives - aussi bien par ses collègues que par les patient-e-s et d'être en mesure de reconnaître ses erreurs comme ses difficultés personnelles. Considérés comme des sujets à part entière, les malades se virent quant à eux octroyer un droit à la parole. Plutôt que de les cantonner dans une position «d'objet passif de soins», on se mit à leur prêter des compétences thérapeutiques, tant pour eux-mêmes que pour leurs congénères. L'octroi d'un tel statut s'accompagna toutefois de nouvelles exigences: on attendit en particulier d'eux qu'ils parviennent à s'affranchir de leur passivité comme de leur attitude oppositionnelle. Le paradoxe est que, pour parvenir à ce résultat, des comportements ouvertement oppositionnels furent tolérés, voire valorisés, ce qui impliquait un changement de posture considérable de la part de l'équipe soignante. Comme le soulignèrent deux psychiatres de l'époque: «La question la plus importante à se poser lorsqu'un malade est agité ou difficile est: Qu'est-ce que ce comportement signifie? et non: Comment pouvons-nous y mettre fin le plus aisément possible? ${ }^{14}$ La permissivité qui résulta de ce repositionnement constitue une autre caractéristique importante de la communauté thérapeutique.

\section{Genèse en temps de guerre}

Instituées rétrospectivement comme fondatrices, les premières expériences se déroulèrent en Grande-Bretagne entre 1941 et 1945, dans le contexte particulier de la Seconde Guerre mondiale ${ }^{15}$. Dans un climat où la question du moral et de la cohésion des troupes se voyait très investie, les services psy-

13 Ainsi que Clark $(1977,559)$ le souligne, l'étendue des domaines discutés collectivement a cependant pu varier considérablement d'une expérience à l'autre: si en certains cas l'équipe thérapeutique et les patient-e-s allèrent jusqu'à élaborer collectivement des décisions concernant les traitements, ces discussions communes furent parfois limitées à l'organisation des loisirs.

14 Le psychiatre belge Claude Bloch cite ici le psychiatre britannique Denis V. Martin: Bloch 1962, 248.

15 Cette première phase est celle qui a été le plus étudiée: Rose 1989; Harrison 2000; Jones 2004; Thalassis 2007. 
chiatriques militaires britanniques furent le théâtre de pratiques novatrices qui impliquèrent des patients souffrant de névroses de guerre.

Dès 1941, le psychiatre Maxwell Jones (1907-1990) ${ }^{16}$ - habituellement salué comme le père de la communauté thérapeutique - introduisit une série de modifications dans sa manière de traiter les patients hospitalisés à Mill Hill, une dépendance du Maudsley Hospital, dans la banlieue de Londres ${ }^{17}$. Responsable d'une unité psychosomatique réunissant une centaine de militaires souffrant de troubles fonctionnels cardiaques, Jones pensa que le meilleur moyen de les amener à admettre qu'ils souffraient de troubles psychosomatiques était de les informer des mécanismes à l'œuvre derrière leurs symptômes. Le nombre des patients ne lui permettant pas de s'entretenir individuellement avec chacun, il organisa des conférences à l'intention de tous. Il constata cependant rapidement que celles-ci n'étaient guère profitables. Aussi décida-t-il de travailler en petits groupes, à raison de trois rencontres par semaine durant un mois ${ }^{18}$. Ayant observé que certains aspects de la vie au sein des divisions étaient abordés par les patients lors de ces réunions, Jones s'intéressa aux «phénomènes sociologiques» ${ }^{19}$ à l'origine des changements d'atmosphère au sein des services. Ces différents constats amenèrent Jones et son équipe à entreprendre dès 1944 des jeux de rôles qui s'apparentaient au psychodrame de Jakob Moreno (1889-1974) - dont ils ignoraient cependant tout alors -, afin d'amener les patients à prendre conscience de leurs problèmes personnels.

La seconde expérience se déroula en deux étapes et sous la houlette de plusieurs psychiatres, au sein d'un hôpital psychiatrique militaire doté de 300-400 lits, situé à Northfield près de Birmingham. Alors que Maxwell Jones put développer son projet durant presque toute la période de la guerre, la première expérience de Northfield, initiée en 1942, fut interrompue après six semaines par la direction de l'hôpital. En dépit de ce désaveu, les psychiatres et psychanalystes Wilfred Bion (1897-1979) et John Rickman (1891-1951) décidèrent de publier un article en commun, paru en 1943 dans The Lancet, afin de rendre compte du travail initié par chacun d'eux à Northfield ${ }^{20}$. On mesure la différence de leur démarche respective lorsqu'on sait que Bion était placé à la tête d'un training ward de 100 patients alors que Rickman entreprit une thérapie de groupe au sein d'une division qui ne comportait qu'une quinzaine de lits. Tous deux cependant entendaient trouver des

16 Maxwell Jones est resté durant toute sa carrière un fervent promoteur de la communauté thérapeutique, voir Millard 1996 et Clark 2005.

17 Jones 1953, 1-15.

18 On trouve plusieurs transcriptions de ces séances de discussion dans Jones 1953, 4-12.

19 Jones 1953, 13.

20 Bion/Rickman 1943. 
moyens pour parvenir à établir un esprit de corps, conduisant chaque patient à contribuer de manière active au bon fonctionnement du groupe. C'est ainsi que Bion, par exemple, réduisit au minimum les exigences relatives au fonctionnement du service et y toléra un désordre certain, afin que la demande de règles de vie en commun émane des patients eux-mêmes plutôt que d'être imposée par les soignants.

Bien que les expériences de Bion et de Rickman aient été interrompues prématurément, le travail de psychothérapie de groupe se poursuivit à Northfield avec l'arrivée en avril 1943 du psychanalyste Heinrich Foulkes (1898-1976). Peu avant la fin de la guerre, le psychiatre et psychanalyste Thomas Main (1911-1990) et Harold Bridger (1909-2005) - professeur de mathématiques avant le début du conflit - initièrent ce qu'on désigne habituellement comme la seconde expérience de Northfield. C'est à son propos que fut pour la première fois employé le terme de «communauté thérapeutique» par Main dans le cadre d'un numéro consacré, en 1946, à la psychiatrie britannique durant la guerre par une revue de psychiatrie américaine d'obédience psychanalytique ${ }^{21}$.

Bien que moins véhémente que ce qu'elle sera au plus fort de l'antipsychiatrie, la critique du fonctionnement habituel des hôpitaux psychiatriques d'alors apparaît clairement dans l'introduction de l'article de Main:

The concept of a hospital as a refuge too often means, however, that the patients are robbed of their status as responsible human beings. Too often they are called «good» or «bad» only according for their obedience, dependency and gratitude. The fine traditional mixture of charity and discipline they receive is a practised technique of removing their initiative as adult beings, and making them «patients». They are less trouble thus to the staff. [...] So, isolated and dominated, the patient tends to remain gripped by the hospital machine even in the games or prescribed occupations which occupy his time between treatments. Within such a setting, health and stability are too often bought at the excessive price of desocialisation. Sooner or later the patient, alone and unsupported, must face the difficult task of returning to the society in which he became unstable, and there regain social integration, and a daily sense of values and purpose. This task is no light for a desocialised man, however healthy he may have become. The design of a hospital as a social retreat also ignores positive therapeutic forces the social support, and emotional opportunities which are granted in spontaneously structured communities. ${ }^{22}$

L'impossibilité matérielle de pratiquer des psychothérapies approfondies au sein des institutions psychiatriques publiques ne constitue pas la seule raison pour Main de s'intéresser au potentiel thérapeutique de la communauté, il lui accorde en effet une pertinence intrinsèque:

It is true that radical individual treatment can free the inner drives of the patient, and make him capable of full and stable social life, but it fails to give him an assured technique for full social participation - he can only learn this from the impact of society itself. Treatment of the neurotic patient, who suffers from a disturbance of social relationships, cannot therefore be

21 Six articles paraissent dans le numéro de mai 1946 du Bulletin of the Menninger Clinic.

22 Main 1946, 66. 
regarded as satisfactory unless it is undertaken within a framework of social reality which can provide him with opportunities for attaining fuller social insight and for expressing and modifying his emotional drives according to the demands of real life. ${ }^{23}$

Dans le reste de son article, Main insiste beaucoup sur les changements de rôle que la mise en place d'une communauté thérapeutique implique tant de la part du psychiatre que du personnel infirmier. Ce thème, également présent d'emblée chez Maxwell Jones, a été abondamment repris par la suite. Notons que dans l'après-guerre ces deux psychiatres furent les seuls à œuvrer au développement de la communauté thérapeutique en tant que courant: Main comme psychanalyste, Jones comme promoteur de la psychiatrie sociale. Bion et Foulkes se tournèrent pour leur part vers le développement de la psychothérapie psychanalytique de groupe. Cela aurait sans doute également été le cas de Rickman s'il n'était pas décédé si rapidement. Quant à Bridger, il mit l'essentiel de ses compétences de psychanalyste au service du management.

\section{Constitution d'un courant}

Au cours de la seconde phase, de 1946 à 1965 environ, la communauté thérapeutique s'est progressivement imposée comme une approche en soi. Un nombre important de publications - des articles mais surtout des monographies - relatant des expériences de plus longue durée fit connaître cette approche aux psychiatres en exercice à cette époque. Par ailleurs, après avoir été expressément présentée comme une approche adaptée aux malades névrotiques durant la guerre, elle fut étendue à d'autres catégories à partir des années 1950.

De 1947 à 1959, Maxwell Jones s'employa à développer la communauté thérapeutique en travaillant avec une population bien particulière de patient-e-s, qualifiés à l'époque de «psychopathes», de «déséquilibrés caractériels», d'«adultes asociaux» ou encore de «délinquants» - une catégorie de malades qui auraient été la «croix» des psychiatres, ces derniers ne sachant que faire pour les traiter ${ }^{24}$. Menée au sein d'une unité mixte de 100 lits $(2 / 3$ d'hommes pour un $1 / 3$ de femmes $)$ - le Belmont Hospital ${ }^{25}-$, cette

23 Main 1946, 66.

24 C'est du moins en ces termes que les patient-es de Jones sont présenté-e-s par un psychiatre suisse ayant fait un voyage d'étude en Grande-Bretagne:Schneider, 1954,465; voir aussi Jones 1954.

25 Rattaché jusqu'en 1959 au Belmont Hospital, un grand hôpital de la banlieue sud de Londres, le service initialement dirigé par Jones a reçu différentes appellations au fil des années: désigné dans un premier temps sous le terme de Industrial Neurosis Unit, il prit le nom de Social Rehabilitation Unit de 1954 à 1959; à partir de 1959, une fois ce service devenu indépendant, Henderson Hospital devint l'appellation définitive. 
expérience a été présentée dans divers articles ${ }^{26}$ et dans deux ouvrages. Le premier, Social Psychiatry, est paru en 1952 en Grande-Bretagne sous la direction de Jones puis en 1953 sous un nouveau titre, The Therapeutic community. A New Treatment Method in Psychiatry, aux USA ${ }^{27}$. Le second fut publié en 1960 en Grande-Bretagne sous le titre de Community as doctor. New Perspectives on a Therapeutic Community ${ }^{28}$. Menée au sein du Belmont Hospital durant les années 1950 par une équipe placée sous la direction de Robert Norman Rapoport, un sociologue américain, cette étude visait à donner de meilleurs fondements théoriques à la communauté thérapeutique.

Quelques années plus tard, le Dr Denis V. Martin ${ }^{29}$ montra pour sa part que la communauté thérapeutique était un mode de prise en charge susceptible d'être appliqué avec profit à l'ensemble des patient-e-s d'un hôpital psychiatrique ordinaire, soit aussi bien aux patient-e-s schizophrènes considéré-e-s comme chroniques qu'aux malades hospitalisé-e-s pour une crise aiguë, ou encore qu'aux névrosé-e-s. Le travail initié dès 1955 par Martin eut également un grand impact car, jusqu'alors, les communautés thérapeutiques avaient toujours été mises sur pied au sein d'unités comprenant une centaine de lits au maximum. Or, après avoir dirigé à titre expérimental une communauté thérapeutique de vingt-sept femmes schizophrènes, Martin parvint à étendre ce modèle à l'ensemble de Claybury, un hôpital de 2200 lits, situé à la périphérie de Londres. Décrivant les étapes de son travail et les difficultés rencontrées, Martin apportait la démonstration avec son livre - Adventure in Psychiatry. Social Change in a Mental Hospital (1962) - que la communauté thérapeutique n'était pas une approche réservée à des petites unités accueillant des patient-e-s ayant un profil bien particulier comme cela avait été le cas jusqu'alors.

Vers la fin des années 1950, un autre psychiatre, David H. Clark, entreprit d'introduire la communauté thérapeutique à Fulbourn, un hôpital psychiatrique de 820 lits, situé près de Cambridge ${ }^{30}$. Quant à Maxwell Jones, ce n'est qu'en 1962, après un séjour de trois ans aux USA, qu'il chercha à implan-

26 Jones 1954; Jones et Rapoport 1955; Jones 1956; Jones et al. 1956.

27 Si Maxwell Jones est présenté comme le premier auteur de ce travail, il fit figurer le nom de six collaborateurs comme coauteurs.

28 La version originale de cet ouvrage n'étant pas disponible, je me suis référée à la traduction française parue en 1974.

29 Malheureusement, je n'ai pas trouvé d'information sur la carrière du Dr Martin.

30 Ayant commencé à pratiquer la communauté thérapeutique dès 1958, le Dr Clark publia en 1964 Administrative Therapy. The role of the Doctor in the Therapeutic Community après avoir séjourné aux USA grâce à une bourse. On trouve plusieurs textes de David Clark sur l'histoire de la communauté thérapeutique et sur sa propre expérience à Fulbourn sur le site web des archives de The Planned Environment Therapy Trust (http://www.pettarchiv.org.uk). 
ter son approche dans un hôpital psychiatrique «ordinaire» de 400 lits, au moment où il prit la direction de l'hôpital écossais de Dingleton, situé à 60 kilomètres d'Edimbourg ${ }^{31}$.

Enfin, le Dr Thomas Main poursuivit quant à lui l'expérience initiée durant la guerre en travaillant essentiellement avec des patient-e-s névrosé-e-s. Dès 1946, il prit en effet la direction d'une clinique psychiatrique privée, le Cassel Hospital, situé dans un comté au sud-est de Londres. Or, bien que cette clinique fût entrée en 1948 dans le National Health Service, elle avait pu conserver l'essentiel de ses caractéristiques antérieures. Elle demeura donc un établissement accueillant des névrosé-e-s, voire parfois aussi la famille de ceux-ci. Mis à part le maintien de son orientation psychanalytique, les moyens matériels pour pratiquer des psychothérapies furent garantis par un taux d'encadrement des patient-e-s particulièrement élevé. En effet, au début des années 1960, près de quatorze psychiatres s'occupaient d'une soixantaine de patient-e- $\mathrm{s}^{32}$. De plus, non seulement la majorité des psychiatres avaient une formation analytique, mais c'était également le cas d'une bonne part du personnel infirmier ${ }^{33}$.

En articulant traitements psychanalytiques individuels et communauté thérapeutique, Main eut surtout un rayonnement important au sein du mouvement psychanalytique, tandis que les travaux de Jones et de Martin montraient, pour leur part, que l'on pouvait pratiquer la communauté thérapeutique sans être psychanalyste ${ }^{34}$. Signalons enfin que c'est seulement à la fin de cette seconde phase que l'emploi du terme «communauté thérapeutique» s'imposa. Auparavant, il s'était vu concurrencer par d'autres notions, comme celles de «psychiatrie sociale» ou de «psychiatrie administrative» ${ }^{35}$. Certains psychiatres américains parlèrent pour leur part de «thérapie par le milieu», ou encore d'«équipe thérapeutique». Au milieu des années 1960, la communauté thérapeutique avait suffisamment gagné en importance en Grande-Bretagne pour susciter la création d'une première association faîtière en 1966 - The Planned Environment Therapy Trust ${ }^{36}$ - puis d'une seconde en

31 Bléandonu 1970, 38. Jones dirigea Dingleton jusqu'en 1969, date à laquelle il partit s'installer définitivement aux USA, il était alors âgé de 62 ans. D'après son collègue David Clark, il tenta d'ouvrir des communautés thérapeutiques dans différents états, mais sans succès. A la fin de sa vie, il aurait développé un vif intérêt pour les questions spirituelles: Clark 2005.

32 Bléandonu 1970, 56.

33 Briggs 1959, 216. D'après Bloor/McKeganey/Fonkert 1988, le rôle thérapeutique des médecins et du personnel infirmier semble toutefois être demeuré clairement distinct (27).

34 Jones a entrepris une psychanalyse et a également été supervisé dans les années 1950, il a toutefois renoncé à devenir membre de la société de psychanalyse britannique: Millard 1996; nous n'avons pas d'informations précises concernant le cursus de Martin, mais certains de ses propos nous conduisent à penser qu'il n'avait pas de formation analytique.

35 Jones utilise les deux notions, Clark la seconde.

36 http://pettrust.org.uk/index.php/Planned_Environment_Therapy_Trust 
1972 - The Association of Therapeutic Communities. ${ }^{37}$ En 1976, cette dernière comptait 30 membres collectifs représentant des institutions ayant adopté le modèle de la communauté thérapeutique, la moitié était établie en GrandeBretagne et le reste à l'étranger et, 10 ans plus tard, leur nombre avait doublé. D'après le sociologue Manning, 17 communautés thérapeutiques étaient en activité en Grande-Bretagne au milieu des années $70^{38}$.

\section{Diffusion en dehors de la Grande-Bretagne}

Sans parler des USA, où de nombreuses communautés thérapeutiques furent fondées, cette approche se diffusa dès les années 1960 en Europe continentale. Fragmentaires, les informations disponibles ne nous permettent toutefois pas de préjuger de sa diffusion effective dans les différents pays.

La communauté thérapeutique semble avoir été bien accueillie aux PaysBas. Une association nationale y fut notamment créée en 1973 à l'initiative de six hôpitaux ${ }^{39}$. De plus, bien acceptée par les instances dirigeantes de la psychiatrie, cette approche n'aurait pas été cantonnée dans une position marginale ${ }^{40}$. Les pays scandinaves lui auraient également réservé un bon accueil ${ }^{41}$. En Allemagne de l'Ouest par contre, où cinq communautés thérapeutiques au moins furent créées au cours des années 1960, la diffusion de ce modèle aurait été entravée - aux dires d'un de ses promoteurs - par le scepticisme conjoint des représentants de la psychiatrie universitaire et des médecins-directeurs des hôpitaux psychiatriques ${ }^{42}$. La communauté thérapeutique suscita également un enthousiasme certain en Italie. Ce modèle fut notamment adopté dès 1968 à Pérouse, deux communautés thérapeutiques furent créées à Milan dans la deuxième moitié des années 1960, ainsi qu'une à Rome et une autre à Venise ${ }^{43}$. Son développement semble toutefois avoir été en partie limité par la querelle entre des représentant-e-s de la communauté thérapeutique accordant une grande importance aux conceptions psychanalytiques et les tenant-e-s de l'antipsychiatrie organisé-e-s dès 1973 au sein du mouvement Psichiatria Democratica, nous y reviendrons ${ }^{44}$.

$37 \mathrm{http}: / /$ www.therapeuticcommunities.org

38 Manning 1989, 30sq. et 53.

39 Dans son ouvrage, Bléandonu (1970, 88-101) rend compte de la visite effectuée en 1967 par le Dr Roger Gentis dans trois communautés thérapeutiques néerlandaises. Voir aussi: Blok 2004.

40 Kennard 1999.

41 Ploeger 1980; Kennard 1999.

42 Ploeger 1980.

43 Legrand 1988, 15; Pietropoli Charmet 1997.

44 Pedrali 1997; Pietropoli Charmet 1997. 
En France, la diffusion de la communauté thérapeutique fut sans doute inhibée par le statut qu'y occupait déjà la "psychothérapie institutionnelle» ${ }^{45}$. En effet, cette approche - également issue d'expériences menées durant la guerre - présente de nombreux points communs avec la communauté thérapeutique. Cette situation n'empêcha toutefois pas le courant britannique de faire l'objet d'une réception en France grâce à un travail éditorial conséquent initié par plusieurs psychiatres, le plus souvent déjà impliqués dans le développement de la psychothérapie institutionnelle. Le Dr Gérard Bléandonu fit ainsi plusieurs conférences et publications suite à deux voyages réalisés en Grande-Bretagne en 1966 et 1967 grâce à une bourse d'études ${ }^{46}$. Après avoir visité plusieurs communautés thérapeutiques néerlandaises, le Dr Roger Gentis présida également à la traduction d'une série de classiques, dont celle du livre du Dr Martin en 1969. Le Dr Paul Sivadon, expert pour l'Organisation Mondiale de la Santé, signa pour sa part une préface enthousiaste pour l'édition française du troisième ouvrage de Maxwell Jones, parue en $1972^{47}$. Une traduction de l'étude sociologique menée par Rapoport dans les années 1950 au Belmont Hospital fut également publiée en 1974 chez François Maspéro, un éditeur militant. Enfin, plusieurs articles de promoteurs de la communauté thérapeutique tels que Main, Jones ou encore Michael Woodbury furent également traduits dans L'Information psychiatrique, le journal du Syndicat des médecins des hôpitaux psychiatriques. En revanche, peu d'expériences concrètes furent entreprises. En 1970, Bléandonu n'avait connaissance que d'un seul projet, mené entre 1965 et 1968, au sein de l'Hôpital psychiatrique de Bassens (Savoie), alors dirigé par le Dr Henri Vermorel $^{48}$.

En Suisse - comme aux Pays-Bas, en Allemagne ou en Italie -, on dénombre au moins sept projets de communautés thérapeutiques initiés entre le début des années 1960 et le milieu des années 1980. Souvent en raison de conflits, la moitié d'entre eux durèrent deux ans à peine. Ce fut le cas du travail mené au sein du pavillon des Cèdres dans la clinique des Rives de Prangins (Vaud) entre 1962 et 1964 environ par le psychiatre américain Michael Woodbury ${ }^{49}$, comme de l'expérience engagée dans l'une des divisions de l'Hôpital psychiatrique universitaire de Genève sous la houlette du Dr Bartold Bierens

45 Chanoît 1995; von Bueltzingsloewen (à paraître); Coffin (à paraître); voir également les chapitres 2 et 3 de la thèse de Henckes 2007.

46 Bléandonu 1967, 1968, 1970, 1971.

47 Jones 1972 (é.o. anglaise 1968).

48 Bléandonu 1970, pp.102-105; Bléandonu s'appuie sur la thèse de médecine de Pierre Dessuant, La communauté thérapeutique. A propos d'une expérience concrète effectuée dans un hôpital psychiatrique, datant de 1969.

49 Woodbury, 1966, ch. VI. 
de Haan entre 1976 et $1977^{50}$. On ignore par contre la durée de deux projets pilotes initiés au sein des institutions psychiatriques universitaires zurichoises $^{51}$. En 1963, le Dr Werner Stoll a mis sur pied une communauté thérapeutique modèle au sein d'une division pour femmes du Burghölzli ${ }^{52}$; ce fut également le cas du Dr Jürg Willi au sein de la division de psychothérapie stationnaire de la policlinique psychiatrique universitaire de Zürich en $1968^{53}$. Les trois autres fonctionnèrent entre 10 et 25 ans. La communauté thérapeutique établie en 1965 au sein de la clinique privée du Schlössli (Zürich) par le Dr Edgar Heim semble avoir fonctionné jusqu'à sa nomination en 1979 à la chaire de psychothérapie de l'Université de Berne, dont il reprit également la direction de la policlinique psychiatrique ${ }^{54}$. De 1966 à 1990, soit durant près de vingt-cinq ans, la communauté thérapeutique fut également une référence centrale pour le Dr Jean Rey-Bellet et son adjoint le Dr Roberto Henking, tous deux placés à la tête de l'Hôpital psychiatrique de Malévoz (Valais) ${ }^{55}$. Au fil des années, la communauté thérapeutique y prit toutefois différentes formes: appliquée initialement dans l'ensemble des pavillons, elle fut surtout mise en œuvre dans celui réservé aux jeunes patient-e-s schizophrènes par la suite. Enfin, à Berne, la communauté thérapeutique de la Soteria accueille toujours des personnes en crise psychotique dans une petite maison d'un quartier résidentiel. Etablie en 1985 sous la forme d'une fondation autonome par le professeur de psychiatrie sociale d'alors, le Dr Luc Ciompi, ce projet semble s'être développé en bonne intelligence avec les institutions psychiatriques officielles du canton ${ }^{56}$.

Mis à part la durée des expériences, on observe également des variations significatives dans le nombre de patient-e-s impliqué-e-s: près de $400 \mathrm{au}$ Schlössli et à Malévoz, entre 10 et 20 dans les autres cas. De même, certaines de ces expériences se développèrent au sein d'institutions publiques (hôpitaux psychiatriques du Valais, de Zurich et de Genève), alors que d'autres

50 Steinauer 1982; Heimberg 2005; je me réfère également à des documents qui se trouvent dans les archives de l'hôpital psychiatrique de Malévoz, ainsi qu'à un échange de correspondance avec le Dr Bierens de Haan au printemps 2008.

51 Selon l'un des relecteurs anonymes de cet article, le Dr Ambros Uchtenhagen aurait également initié une communauté thérapeutique au début des années 1970 en collaboration avec l'association «Die Alternative» qui s'occupe de la prise en charge des personnes ayant des problèmes de drogues, sans avoir pour autant écrit sur cette expérience.

52 Germann 2007, 233.

53 Kind 2000, 546.

54 Heim 1978. Heim a également signé en 1976 la préface de la traduction allemande d'un des ouvrages de Maxwell Jones.

55 Les renseignements concernant la communauté thérapeutique en Valais sont tirés d'une recherche en cours reposant sur des archives et des sources orales.

56 Aebi/Ciompi/Hansen 1993; Ciompi/Hoffmann/Broccard 2001; Ciompi 2005; http://www. soteria.ch 
eurent pour cadre des cliniques ou des fondations privées (le Schössli, les Rives de Prangins et la Soteria). Enfin, si l'antipsychiatrie semble avoir constitué une référence positive à Genève et à la Soteria, il est probable que seule cette dernière ait opté pour l'usage des prénoms et l'abandon de la blouse blanche, une suppression des signes de hiérarchie entre personnel et soignant pourtant chère à Maxwell Jones.

\section{Radicalisation et remise en cause}

Au cours des années 1960, parallèlement à sa diffusion au sein des pays occidentaux, la communauté thérapeutique a également été investie par le mouvement antipsychiatrique et cela aussi bien en Italie qu'en GrandeBretagne. En raison de la notoriété acquise par l'antipsychiatrie et de son autoreprésentation comme rupture radicale, peu de gens savent toutefois aujourd'hui que la communauté thérapeutique a constitué le cadre de travail initial aussi bien de Franco Basaglia (1924-1980), de Ronald Laing (19271989) que de David Cooper (1931-1986).

En Grande-Bretagne, la communauté thérapeutique de Kingsley Hall est considérée comme l'expérience fondatrice du mouvement antipsychiatrique $^{57}$. Mené de 1965 à 1970, ce projet se trouve habituellement associé au nom de Ronald Laing, quand bien même d'autres psychiatres s'y impliquèrent. Si plusieurs acteurs sociaux de l'époque ont présenté Kingsley Hall comme une forme particulière de communauté thérapeutique ${ }^{58}$ - dans laquelle le système des réunions régulières avait cédé la place à une organisation plus libertaire et où des comportements beaucoup plus transgressifs étaient tolérés ${ }^{59}$-, sa notoriété provient essentiellement de son statut de projet alternatif radical.

Le travail engagé entre 1962 et 1966 par le Dr David Cooper dans le cadre de la «Villa 21» - une petite division située dans un grand hôpital psychiatrique de 2000 lits - eut également un retentissement certain. Dans son ouvrage Psychiatry and Anti-Psychiatry paru en 1967, Cooper la présenta comme la première expérience antipsychiatrique menée en Grande-Bre-

57 A ce jour, l'expérience de Kingsley Hall n'a pas fait l'objet d'une étude historique, aussi ce qu'on en sait repose essentiellement sur l'ouvrage relatant «le voyage à travers la folie» de Mary Barnes de son propre point de vue et de celui du psychiatre Joseph Berke qui l'a «accompagnée»: Barnes/Berke, 1971.

58 Bleandonu 1968, 749; Barnes/Berke 1971; Clark 1977, 555; Bloor/McKeganey/Fonkert 1988, 35 sq.

59 Clark, 1977, 555; pour une analyse des pratiques et des discours des promoteurs de la communauté thérapeutique des années 1950 en regard de celles des antipsychiatres, voir Fussinger (à paraître). 
tagne. Pourtant, la description qu'il en donne nous montre que le fonctionnement de cette communauté thérapeutique accueillant une vingtaine de jeunes hommes, diagnostiqués schizophrènes, ressemblait à bien des égards à ce qui avait été décrit par un Maxwell Jones ou un Denis Martin. Si Cooper mentionne les emprunts faits à la communauté thérapeutique, il n'a de cesse de souligner le caractère unique et singulier de son expérience à la Villa $21^{60}$. Et force est de constater que cette lecture s'est rapidement imposée.

L'analyse que fit Cooper de la fin de l'expérience de la Villa 21 eut également une incidence sur l'histoire de la communauté thérapeutique. Les conflits survenus avec la direction de l'hôpital psychiatrique ayant été à l'origine de cet échec, Cooper affirma que de véritables communautés thérapeutiques ne pouvaient se développer qu'en dehors de la psychiatrie officielle, sous forme de projets alternatifs autonomes, comme celui de Kingsley Hall. Si ce point de vue ne s'est pas imposé comme la nouvelle doxa parmi les représentant-e-s de ce courant, la question des avantages et des inconvénients respectifs des deux solutions fut par contre régulièrement débattue ${ }^{61}$.

Après les années 1960, pas plus Laing que Cooper ne se distinguèrent par la création de nouvelles communautés thérapeutiques. Néanmoins, ce modèle continua à être promu par deux associations ayant les liens forts avec le mouvement antipsychiatrique: la Philadelphia Association ${ }^{62}$ et l'Arbors Association $^{63}$. Parmi les héritages de Kingsley Hall, il faut encore mentionner la communauté thérapeutique créée par le Dr Loren R. Mosher (19332004) dans la Baie de San Fransisco (USA). En activité de 1971 à 1983 sous le nom de «Soteria House», ce projet se caractérise essentiellement par la volonté de traiter les crises psychotiques sans recourir aux neuroleptiques ${ }^{64}$.

Du côté anglo-saxon, l'appropriation du modèle de la communauté thérapeutique par la mouvance antipsychiatrique s'est donc surtout traduite par une radicalisation de certaines pratiques ainsi que par une remise en cause systématique de tous les traitements somatiques ${ }^{65}$. En Italie, les rapports du

60 Cooper 1970 (é.o. anglaise 1967), 13.

61 Clark 1977, 554.

62 Fondée en 1964 par un petit groupe de psychiatres, parmi lesquels figuraient notamment Laing et Cooper, cette association a poursuivi ses activités après la fermeture de Kingsley Hall dont elle avait permis la mise sur pied; elle est toujours en activité aujourd'hui: http://www.philadelphia-association.org.uk

63 Cette association a été fondée en 1970 par des psychiatres qui s'étaient vu refuser l'accès à la Philadelphia Association, parmi lesquels se trouvait Joseph Berke (Burston 1996, 90-92), elle est également toujours en activité: http://www.arbourscentre.org.uk

64 Mosher 1991; Mosher 1999; Mosher/Hendrix/Fort, 2004; Bola/Mosher 2005. Plus globalement, sur la question du rapport des promoteurs de la communauté thérapeutique avec les traitements biologiques, notamment les psychotropes, voir Fussinger (à paraître).

65 Fussinger (à paraître). 
mouvement antipsychiatrique avec la communauté thérapeutique prirent une tournure différente. Les débuts de l'antipsychiatrie dans ce pays sont habituellement assimilés au travail mené à partir de 1962 par Basaglia à Gorizia, un hôpital psychiatrique de 400 lits situé à la frontière italo-yougoslave. Or, l'essentiel des efforts porta au départ sur la mise en œuvre des principes de la communauté thérapeutique, tels qu'ils s'étaient développés durant les années 1950 en Grande-Bretagne ${ }^{66}$. Ce n'est que dans un second temps que Basaglia et ses collaborateurs formulèrent ouvertement des réserves envers ce modèle, comme le montrent les propos du psychiatre Lucio Schittar parus dans L'Institution en négation - un ouvrage collectif édité en 1968 sous la direction de Basaglia en Italie.

Le modèle lewinien de résolution des conflits sociaux a été appliqué à la communauté thérapeutique comme il l'avait été à l'administration et à l'industrie pour accroître leur efficacité. Selon lui «la participation» de chacun permet de réduire tous les conflits par la discussion; la <manipulation〉 de groupe permet d'émousser les contrastes les plus saillants par une attitude tolérante et compréhensive; la discussion de groupe oriente les efforts des participants, sous la conduite d'un leader éclairé, vers le <bon> objectif commun: qu'il s'agisse du déroulement ordonné du processus administratif, d'une production régulière, ou de la guérison-réhabilitation-intégration du malade mental. [...] Le cycle semble clos. Ce qui était né comme une exigence de renouveau fondamental des institutions psychiatriques se révèle tout au plus, dans la pratique et en théorie, comme un nouveau type d'institution, plus moderne, plus efficace, mais où les rapports de pouvoir sont restés apparemment les mêmes. $\mathrm{La}$ 〈troisième révolution psychiatrique〉 ne serait donc qu'une adaptation tardive des modalités de contrôle social du comportement pathologique aux méthodes de production perfectionnées au cours des quarante dernières années par les sociologues et les techniciens de la communication de masse. Sous prétexte d'assainir les structures foncièrement déshumanisantes de l'asile d'aliénés, sociologues et psychologues de l'organisation semblent avoir trouvé le moyen d'appliquer au domaine de la psychiatrie institutionnelle les techniques (avant tout, des techniques de groupe) qui se sont révélées si efficaces dans la gestion de l'économie néocapitaliste, sans toucher cependant au pouvoir oppressif de la société67.

Ces critiques ne furent toutefois pas à l'origine de la fin de la communauté thérapeutique à Gorizia. Cette expérience cessa suite aux démissions successives de Basaglia en 1968, puis du reste de son équipe en 1972. En effet, d'importants conflits avaient surgi avec les autorités locales, encore attisés par l'assassinat commis par un patient de l'hôpital psychiatrique en congé dans sa famille. En dépit de ces réserves envers la communauté thérapeutique, Basaglia y recourut également lorsqu'il prit la direction de l'hôpital psychiatrique de Trieste en $1972^{68}$. Assez rapidement toutefois, la fermeture

66 Basaglia 1966. Dans cet article, Basaglia se réfère explicitement à l'ouvrage de Denis V. Martin et aux travaux de Maxwell Jones.

$67 \mathrm{La}$ «troisième révolution psychiatrique» évoquée dans cette citation renvoie au statut revendiqué pour la «psychiatrie sociale» par les psychiatres réformateurs des années 1950, notamment par Maxwell Jones. Ne parlant pas l'italien, je me suis référée à la traduction française: Schittar 1970 (é.o. 1968) 148sq. Voir également dans cet ouvrage la contribution commune de Pirella/Slavich/Jervis/Basaglia, ainsi que celle de Slavich.

68 Rotelli 1982. 
des hôpitaux psychiatriques apparut comme une mesure plus pertinente que les efforts déployés pour leur transformation. Le modèle de la communauté thérapeutique se vit dès lors supplanté en Italie par la proposition de transférer l'ensemble de la prise en charge des patient-e-s psychiatriques dans des services ambulatoires établis dans la cité. Porté par une pluralité d'acteurs sociaux, ce projet se vit renforcé par la création en 1973 de l'Association Psichiatria Democratica. Ce mouvement joua en effet un rôle important dans le processus ayant conduit à l'adoption, en mai 1978, de la loi 180 qui prévoyait la fermeture des hôpitaux psychiatriques en Italie. Concrètement, cette loi interdisait l'admission de nouveaux patients dans les hôpitaux psychiatriques. En lieu et place, chaque région devait créer un service extrahospitalier de santé mentale à même de répondre à l'ensemble des besoins de la population (prévention, cure et réhabilitation). Lorsque des hospitalisations s'avéraient indispensables, celles-ci devaient se faire dans des services de diagnostic et de traitements spécifiques établis au sein des hôpitaux généraux, dont la taille était limitée à 15 lits et dans lesquels la durée du séjour était limitée à sept jours, sauf prolongation explicite dûment justifiée.

$\mathrm{Si}$, au sein du courant Psichiatria Democratica, la communauté thérapeutique a parfois été considérée comme une transition nécessaire préparant une prise en charge entièrement extrahospitalière, elle s'est surtout trouvée critiquée comme une solution «technique» venant masquer l'exclusion sociale des malades. L'impact de l'antipsychiatrie sur la carrière de la communauté thérapeutique s'avère donc difficile à apprécier. Présenté comme une alternative progressiste au fonctionnement traditionnel des hôpitaux psychiatriques, ce courant a suscité un intérêt certain au sein des mouvements sociaux contestataires des années 1960-1970. En même temps, les critiques adressées à son endroit ont également amené les militant-e-s à s'en désintéresser. Enfin, il n'est pas impossible que la popularité de la communauté thérapeutique au sein de la contre-culture ait joué en sa défaveur auprès des représentant-e-s de la psychiatrie officielle.

\section{Institutionnalisation et déclin}

Durant les deux dernières décennies du $20^{\text {ème }}$ siècle, les communautés thérapeutiques encore en activité entrèrent, selon le sociologue Manning, dans une phase de «professionnalisation et de routine» ${ }^{69}$. Pour le psychiatre David Clark toutefois, la communauté thérapeutique cessa d'être «à la mode» en

69 Manning 1989. 
Grande-Bretagne dès les années 1970. Aussi, la phase d'institutionnalisation tend ici à se confondre avec le mouvement de déclin ${ }^{70}$. Les difficultés rencontrées furent de plusieurs ordres.

Souvent tributaire du charisme d'un leader, la communauté thérapeutique - de par la nature même de son fonctionnement - semble avoir été un projet difficile à maintenir sur la durée. Basée sur une confrontation collective continue, suscitant bien évidemment de nombreuses crises, l'expérience se révéla épuisante pour le personnel. A ces difficultés internes s'ajoutèrent des rapports conflictuels avec le milieu environnant et cela tant au sein des hôpitaux psychiatriques qu'en dehors de ceux-ci. Si dans les années 1960-1970, l'espoir d'un changement social radical très proche a réduit la portée de ces obstacles, le reflux de l'engagement militant à partir des années 1980 n'est pas resté sans incidences pour le courant de la communauté thérapeutique; il devint notamment rare de trouver des personnes prêtes à s'engager bénévolement dans un tel projet ${ }^{71}$.

Par ailleurs, de l'avis des premiers intéressés, les réformes que connurent la plupart des systèmes de santé européens dans ces années-là se révélèrent très peu propices au maintien des communautés thérapeutiques ${ }^{72}$ : avec le développement des prises en charge ambulatoires, les séjours de longue durée dans une institution thérapeutique assurant un hébergement étaient de moins en moins tolérés ${ }^{73}$. Enfin, si quelques commentateurs estiment qu'un certain déficit en matière de conceptualisation pesa défavorablement sur le développement de la communauté thérapeutique ${ }^{74}$, il fut surtout difficile, dans ce cas comme dans bien d'autres, d'apporter la «preuve scientifique de l'efficacité de cette approche» ${ }^{75}$. Or, on le sait, de telles «preuves» furent exigées de plus en plus systématiquement au cours des deux dernières décennies du $20^{\text {ème }}$ siècle.

En réponse à ces difficultés, on observe durant cette période des efforts d'institutionnalisation. Ainsi, l'Association of Therapeutic Communities fonda en 1980 The International Journal for Therapeutic and Supportive Organizations. A l'instar de ce qui se pratiquait dans le domaine des psychothérapies, l'idée de formaliser un cursus de formation fut avancée, sans grand succès toutefois. Ainsi, un représentant de la communauté thérapeu-

70 Clark 1977.

71 Il semblerait notamment que tous les psychiatres qui travaillèrent à Kingsley Hall le firent de manière bénévole. En Italie aussi, des bénévoles s'engagèrent dans les structures psychiatriques alternatives créées pour la plupart dans le nord du pays.

72 Manning 1989, 48; Kennard 1999, 243.

73 Notons toutefois que certains hôpitaux de jour adoptèrent le modèle de la communauté thérapeutique.

74 Manning 1989.

75 Clark 1977, 557sq. 
tique se demandait en 1999 quelle stratégie était envisageable pour permettre à ce courant de survivre. Si, en Grande-Bretagne, la communauté thérapeutique semble demeurer une référence vivante aujourd'hui encore, le travail de mémoire initié au sein de ce mouvement dénote une claire conscience de son déclin ${ }^{76}$. Ainsi, certaines associations britanniques commencent à dresser une liste des communautés thérapeutiques disparues tandis que d'autres lancent des appels à la constitution d'archives à même de documenter à l'avenir l'histoire de cette approche.

\title{
En guise de conclusion
}

De l'avis même de certains de ses représentants, le modèle de la communauté thérapeutique semble avoir rencontré des difficultés à s'adapter aux transformations survenues au cours de deux dernières décennies du $20^{e}$ siècle au sein du champ psychiatrique ${ }^{77}$. Doit-on pour autant conclure que cette approche s'est peu à peu éteinte sans laisser de traces? Lorsqu'en 1977, Clark établit le bilan des trente premières années d'existence de ce courant, il lui paraît pourtant évident que bon nombre des pratiques alors en vigueur dans les institutions psychiatriques britanniques lui sont redevables:

\begin{abstract}
A number of components of the therapeutic community method have become part of standard psychiatric practice. The majority staff, even in general hospitals, do not now wear uniform. A style of openness, first-naming and decision-making by consensus is widely used. Although <patient government is not so widely used, ward meetings for the sharing of news and the planning of activities are very widespread. Group therapy, sociodrama and psychodrama, conducted by others than doctors and professional psychotherapists, is now quite common, and the number of professional staff experienced in group work is steadily rising. ${ }^{78}$
\end{abstract}

Un tel constat vaut-il pour l'ensemble des pays occidentaux? Les pratiques qui semblaient bien établies dans les années 1970 ont-elles perduré? Si la question reste ouverte en ce qui concerne l'abandon de la blouse blanche ou l'usage des prénoms, il est indéniable que la communauté thérapeutique a contribué à une réelle transformation de la culture interne aux institutions psychiatriques. En refusant le système hiérarchique pyramidal dominant dans les hôpitaux psychiatriques du milieu du $20^{\mathrm{ème}}$ siècle et en exigeant

76 Kennard 1999,244. Certaines associations britanniques ont par exemple produit une liste des communautés thérapeutiques disparues, une démarche qui se voit encadrée par des mesures visant à créer des archives.

77 On notera que la situation semble différente en ce qui concerne la prise en charge de la toxicomanie, domaine dans lequel le modèle de la communauté semble une référence prégnante aujourd'hui encore. Ainsi l'European Federation of Therapeutic Communities, fondée en 1978 et toujours en activité aujourd'hui, se focalise sur ce type de prise en charge (http://www.eftc-europe.com).

78 Clark 1977, 563. 
une communication plus horizontale, ce courant a en effet fait le lit de pratiques parfaitement banales aujourd'hui telles que les réunions d'équipe ou les colloques réunissant soigné-e-s et soignant-e-s. Aussi, bien que la communauté thérapeutique soit une innovation plus difficile à cerner que d'autres, elle a toute sa place dans l'histoire de la psychiatrie de la seconde moitié du $20^{\text {ème }}$ siècle et l'on ne peut que souhaiter que de nouvelles études historiques, basées notamment sur des sources orales, viennent éclairer la richesse et la diversité d'expériences fortement marqués par leur contexte singulier.

\section{Bibliographie}

Aebi, Elisabeth/Luc Ciompi/Hansen Hartwig (Hrsg.), Soteria im Gespräch. Über eine alternative Schizophreniebehandlung (Bonn 1993)

Basaglia, Franco, «La 〈communauté thérapeutique〉 base d'un service psychiatrique, réalités et perspectives», L'information psychiatrique 42 (1966) 413-428

Barnes, Mary/Joseph Berke, Mary Barnes. Un voyage à travers la folie (Paris 1973; é.o. anglaise 1971)

Barton, Russel, La névrose institutionnelle (Paris 1969; é.o. anglaise: Institutional Neurosis 1959)

Bion, Wilfrid R./John Rickman, "Intra-group tensions in therapy. Their study as the task of the group", The Lancet (1943) 678-681

Bleandonu, Gérard, «A la recherche de la communauté thérapeutique britannique (à propos d'un voyage d'étude)», L'information psychiatrique (1967) 1247-1266

- «La communauté thérapeutique (à propos d'une bourse d'étude du Conseil de l'Europe)», L'information psychiatrique 44 (1968) 739-751

- Les communautés thérapeutiques (Paris 1970)

- «Les communautés thérapeutiques», Revue pratique de psychologie de la vie sociale et d'hygiène mentale 1 (1971) 73-88 (suivi d'un débat où sont reproduites notamment les interventions des $\mathrm{D}^{\text {rs }}$ Tosquelles, Oury 89-99)

Bloch, Claude, «Aspects de l'assistance psychiatrique en Grande-Bretagne», L'information psychiatrique 38 (1962) 233-248, 355-363, 435-439

Blok, Gemma, Baas in eigen Brein. "Antipsychiatrie» in Nederland, 1965-1985 (Amsterdam 2004)

Bloor, Michael. J., "Social control in the therapeutic community: re-examination of a critical case", Sociology of Health and Illness 8 (1986) 305-324

Bloor, Michael J./J. Dick Fonkert, "Reality construction, reality exploration and treatment in two therapeutic communities", Sociology of Health and Illness 4 (1982) 125-140

Bloor, Michael/Neil McKeganey/Dick Fonkert, One foot in Eden: a sociological study of the range of therapeutic community practice (London 1988)

Bola, R. John/Loren R. Mosher, “The Legacy of Kingsley Hall I: Treatment of Acute Psychosis Without Neuroleptics: Two-Year Outcomes From the Soteria Project", in: Salman Raschid (ed.)/R. D. Laing, Contemporary Perspectives (London 2005) 249-272

Briggs, Dennie Lynn, "Social Psychiatry In Great Britain”, The American Journal of Nursing 59 (1959) 215-220

Busfield, Joan, Managing madness: changing ideas and practice (London 1986)

von Bueltzingsloewen, Isabelle, «Soigner l'individu et/ou le collectif? Les premières expériences de psychothérapie sociale dans les hôpitaux psychiatriques français (1945-1952)», dans: Catherine Fussinger/Vincent Barras (éds), Pour une histoire croisée de la psychothérapie comme pratique professionnelle au $20^{e}$ siècle en Europe (Lausanne, à paraître)

Burston, Daniel, The Wing of madness. The life and work of R. D. Laing (Cambridge [Mass.]/ London) 1996 
Campling, Penelope/Rex Haigh Rex (eds), Therapeutic Communities. Past, Present and Future (London/Philadelphia 1999)

Chanoît, Pierre F., La psychothérapie institutionnelle (Paris) 1995

Ciompi, Luc, "The Legacy of Kingsley Hall II: The Soteria-Concept. Theoretical Bases And Practical 13-Year Experience With Milieu-Therapeutic Approach to Acute Schizophrenia (The Swiss Soteria Bern Project", in: Salman Raschid (ed.), R. D. Laing. Contemporary Perspectives (London 2005) 272-295

- /Roger Hoffmann/Michel Broccard (Hrsg.), Wie wirkt Soteria? Eine atypische Psychosenbehandlung kritisch durchgeleutet im Gespräch (Bern 2001)

Clark, David H., Administrative Therapy. The Role of the Doctor in the Therapeutic Community (London 1964)

- "Therapeutic Community", British Journal of Psychiatry 131 (1977) 553-564

- "Therapeutic Community Memories: Maxwell Jones", Planned Environment Therapy Trust Archive and Study Centre (2005; disponible sur le site: http://www.pettarchiv.org.uk/ pubs-dhclark-maxjones.htm).

Coffin, Jean-Christophe, «Georges Daumezon et la psychothérapie institutionnelle française (1945-1970). Retour sur un article fondateur et ses usages», dans: Catherine Fussinger/ Vincent Barras (éds), Pour une histoire croisée de la psychothérapie comme pratique professionnelle au $20^{e}$ siècle en Europe (Lausanne, à paraître)

Cooper David, Psychiatrie et anti-psychiatrie (Paris, 1970; é. o. anglaise 1967)

Crossley, Nick, "R. D. Laing and the British Anti-Psychiatry Movement: A Socio-Historical Analysis", Social Science \& Medicine 47 (1998) 877-889

Fussinger, Catherine, "Beyond or Before Antipsychiatry. Towards a Historical Reapprehension of the "Therapeutic Community", History of Psychiatry (à paraître 2011)

Grob, Gerald, The mad among us: a history of the care of America's mentally ill (Cambridge [Mass.]/London) 1994

Harrison, Tom/Rickman Bion, Foulkes and the Northfield experiments: advancing on a different front (London 2000)

Heim, Edgar, «Phasenweise Integration eines Millieukonzeptes», in: Edgar Heim (Hrsg.), Milieu-Therapie. Erlernen sozialer Verhaltensmuster in der psychiatrischen Klinik (Bern 1978) $31-53$

Heimberg, Charles, «La contestation de l'institution psychiatrique et sa dimension politique: Bel-Air dans l'œil du cyclone (1977-1983)», Cahiers d'histoire du mouvement ouvrier 21 (2005) 187-195

Henckes, Nicolas, Le nouveau monde de la psychiatrie française. Les psychiatres, l'Etat et la réforme des hôpitaux psychiatriques de l'après-guerre aux années 1970, Thèse de doctorat en sociologie, Ecole des hautes Etudes en Sciences Sociales de Paris (Paris 2007)

Germann, Urs, «Arbeit als Medizin: Die <Aktivere Krankenbehandelung〉 1930-1960», in: Marietta Meier Marietta/Brigitta Bernet/Roswitha Dubach/Urs German (Hrsg.), Zwang zur Ordnung: Psychiatrie im Kanton Zürich 1870-1970 (Zürich 2007) 195-233

Jones, Maxwell, The Therapeutic Community. A new Treatment Method in Psychiatry (New York 1953)

- «Delinquency and Crime. A Challenge to Psychiatry», The Lancet (1954) 1277-1278)

- «The Concept of Therapeutic community», American Journal of Psychiatry 112 (1956) 647650

- Au-delà de la communauté thérapeutique, avec une préface de Paul Sivadon (Villeurbanne 1971; é. o. anglaise 1968)

- /Robert N. Rapoport., «Administrative and social psychiatry», The Lancet (1955) 386-388

Jones, Maxwell/B. A. Pomryn/Eileen Skellern, "Work Therapy", The Lancet (1956) 343-344

Jones, Maxwell et al., The Therapeutic Community. A New Treatment Method in Psychiatry (New York 1953; é. o. anglaise 1952)

Jones, Kathleen, A History of The Mental health Services (London/Boston 1972)

Jones, Edgar, "War and the Practice of Psychotherapy: the UK Experience 1939-1960", Medical History 48 (2004) 493-510

Kennard, David, "Therapeutic Communities in Europe. One modality with Different Models", in: Penelope Campling/Rex Haigh (eds), Therapeutic Communities. Past, Present and Future (London/Philadelphia 1999) 235-245 
Kind, Hans, «Die psychiatrische Poliklinik im Universitätspital», Zürcher Spitalgeschichte, vol. 3, (Zürich 2000) 542-547

Legrand, Michel, «Présentation: L'expérience de Pérouse», dans: Carolo Brutti/Francesco Scotti (éds), Réussir la psychiatrie alternative. L'expérience de Pérouse (Paris 1988; é.o. italienne 1981) 7-21

Main, Tom F., "The Hospital as a Therapeutic Institution", Bulletin of the Menninger Clinic 10 (1946) 66-70

- "The concept of the therapeutic community: variations and vicissitudes", in: Maclom Pines (ed.), The evolution of group analysis (London/Boston 1983) 197-217

Manning, Nick, "The Case of the Therapeutic Community", Journal of Social Policy 5 (1976) 265-279

- The Therapeutic Community Movement: Charisma and Routinisation (London/New York 1989)

Martin, Denis V., Aventure en psychiatrie. Evolution sociologique d'un hôpital (Paris 1969; é. o. anglaise 1962)

Millard, David Walter, "Maxwell Jones and the Therapeutic Community”, in: Hugh Freeman/ German E. Berrios (eds), 150 years of British Psychiatry, Vol. II:The Aftermath (London 1996) 581-604

McKeganey, Nick P./Michael J. Bloor, "Teamwork, information control and therapeutic effectivness: a tale of two therapeutic communites", Sociology of Health and Illness 9 (1987) $154-178$

Mosher, Loren R., "Soteria: A Therapeutic Community for Psychotic Persons", International Journal of Therapeutic Communities 12 (1991) 53-67

- "Soteria and Other Alternatives to Acute Psychiatric Hospitalization. A personal and Professional Review", The Journal of Nervous and Mental Disease 187 (1999) 142-149

- /Voyce Hendrix/Deborah C. Fort, Soteria. Trough Madness to Deliverance (Philadelphia 2004)

Pedriali, Enrico, "Italian therapeutic communities: From historical analysis to hypotheses for changes", Therapeutic Communities 18 (1997) 3-13

Pietropolli Charmet, Gustavo, "Reflections on the experience of therapeutic communities in Italy", Therapeutic Communities 18 (1997) 123-131

Pirella, Agostino/Antonio Slavich/Giovanni Jervis/Franco Basaglia, «Réunion d'équipe du 27 novembre 1967», dans: Franco Basaglia (éd.), L'institution en négation. Rapport sur l'hôpital psychiatrique de Gorizia (Paris 1970; é.o. italienne 1968) 151-154

Ploeger, Andreas, "The therapeutic Community in the Federal Republic of Germany in the past, at Present and in the Future", International Journal of Therapeutic Communities 1 (1980) $75-79$

Rapoport, Robert N., La communauté thérapeutique (Paris 1974; é.o. anglaise 1960)

Rose, Nikolas, Gouverning the soul. The shaping of private self (London/New York 1989)

Rotelli, Franco «L'application de la loi à Trieste», Transitions. Revue internationale du changement psychiatrique et social 17 (1982) 63-71

Roudinesco Elisabeth/Michel Plon, Dictionnaire de la psychanalyse (Paris 1997)

Schittar Lucio, «L'idéologie de la communauté thérapeutique», dans: Franco Basaglia (éd.), L'institution en négation. Rapport sur l'hôpital psychiatrique de Gorizia (Paris 1970; é.o. italienne 1968) 141-150

Schneider, Pierre-Bernard, «Au sujet d'une expérience en cours: Le traitement des déséquilibrés caractériels d'après Maxwell Jones», Schweizer Archiv für Neurologie und Psychiatrie 73 (1954) 463-469

Sharp Victor, Social control in the therapeutic community (Farnborough/Lexington [Mass.] 1975)

Slavich, Antonio, «Mythe et réalité de l'autogouvernement», dans: Franco Basaglia (éd.), L'institution en négation. Rapport sur l'hôpital psychiatrique de Gorizia (Paris 1970; é.o. italienne 1968) 155-169

Spandler, Helen, Asylum to Action. Paddington Day Hospital, Therapeutic Communities and Beyond (London 2006)

Stanton, Alfred/Morris Schwartz, The Mental Hospital. A study of Institutional Participation in Psychiatric Illness and Treatment (New York 1954)

Steinauer, Jean, Le fou du Rhône: documents sur la crise psychiatrique genevoise (Genève 1982)

Gesnerus 67 (2010) 
Thalassis, Nafsika, "Soldiers in Psychiatric Therapy: The Case of Northfield Military Hospital 1942-1946", Social History of Medicine 20 (2007) 351-368

Wiley, Juniper, "Role blurring in a holistic therapeutic community. An Intermediary Response to Institutionalization and Deinstitutionalization", Journal of Contemporary Ethnography 178 (1986) 3-39

Woodbury, Michael, «L'équipe thérapeutique. Principes de traitement somato-psycho-social des psychoses», L'Information psychiatrique 42 (1966) 1035-1142

240 Gesnerus $67(2010)$ 